\title{
Intelligent Transport Systems: Co-Operative Systems (Vehicular Communications)
}

\author{
Panagiotis Lytrivis and Angelos Amditis \\ Institute of Communication and \\ Computer Systems (ICCS) \\ Greece
}

\section{Introduction}

The term Intelligent Transport Systems (ITS) is used to illustrate the application of information and communication technologies in the transport domain. The intention of ITS is to enhance road safety and traffic efficiency, minimize environmental impact and in general maximize the benefits for the road users (Zhou et al., 2010; Popescu-Zeletin et al., 2010; Hartenstein \& Laberteaux, 2010).

In turn, Co-operative Systems are the most promising technology within the ITS framework. The word "co-operative" indicates that vehicles are collaborating with each other and with the infrastructure, exploiting wireless communications, in order to increase their awareness about the road environment. There are two types of communication in co-operative systems, namely vehicle-to-vehicle (V2V) and vehicle-to-infrastructure (V2I).

The scope of this chapter is to highlight the significant role of vehicular communications in future ITS. Standalone sensors and sensor systems can support the drivers in certain cases (e.g. maintain a safe speed and safe distance from the vehicle ahead, avoid a possible rearend collision etc.) but are not sufficient enough. Vehicles exchanging real-time messages and sharing information about the perception of the road environment could significantly extend the benefits of the abovementioned standalone systems and also satisfy the requirements of a large number of applications (see Figure 1).

Over the past years significant efforts have been performed for the bandwidth allocation and the standardization of vehicular communications worldwide. The Federal Communication Commission (FCC) decided the allocation of a frequency spectrum for vehicular applications. In Europe under the European Commission Decision 676/2002/EC the radio spectrum dedicated to ITS is in the $5.8 \mathrm{GHz}$ frequency band. ETSI and CEN have formed working groups and technical committees dedicated to the ITS domain.

Although the benefits from the use of co-operative systems in transport are numerous there are also some difficulties. Some of the concerns are the following: wide uptake of such systems, market penetration, standards finalization and consensus among different standardization organizations, all the inherent problems of wireless technologies (multipath propagation, security issues etc.). 


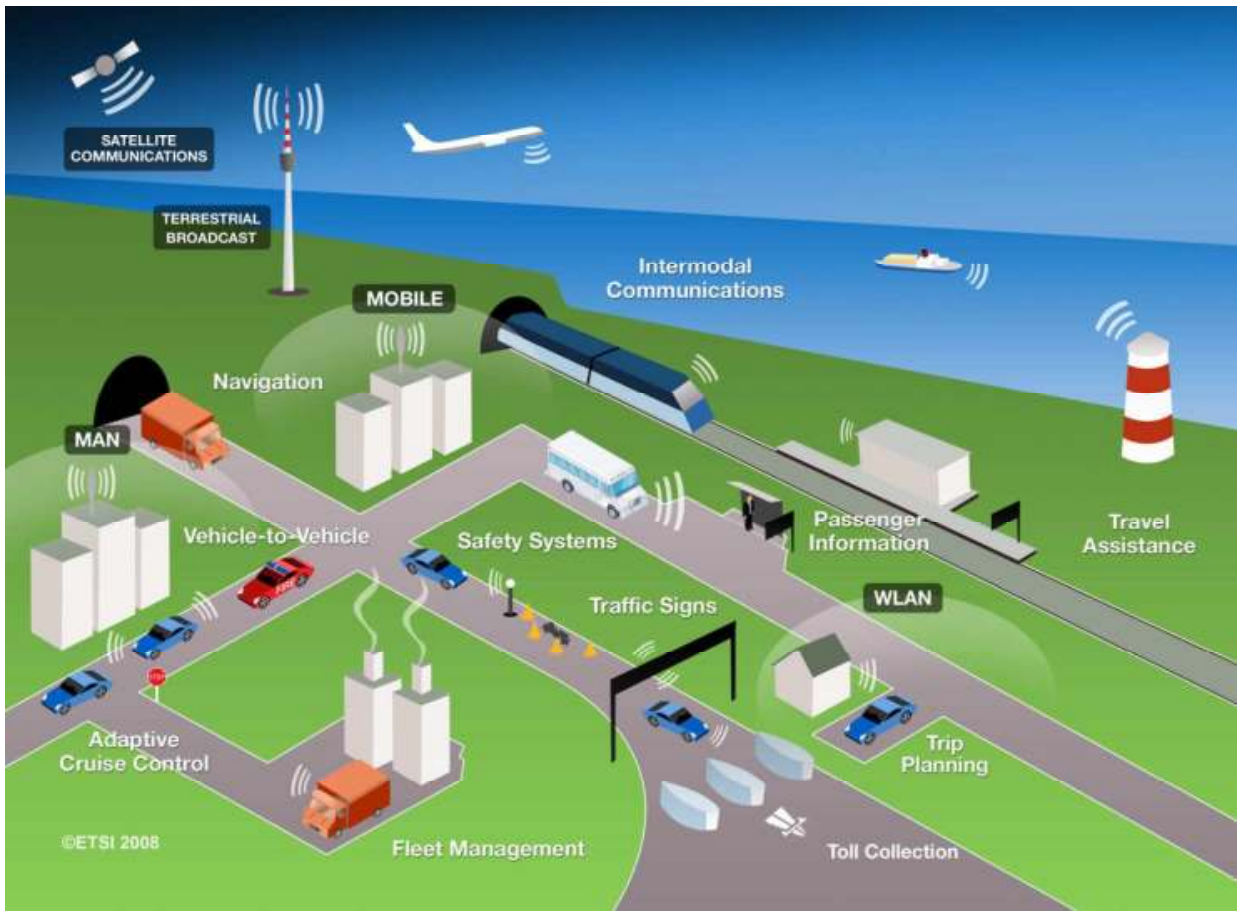

Fig. 1. Indicative ITS applications (ETSI, 2011).

The remainder of this chapter is organized as follows. In the next section, the architecture of co-operative systems is described. In the following wireless technologies used within the cooperative systems framework are outlined. The applications of vehicular networks and their corresponding categories are highlighted. Emphasis is given on hot research topics concerning co-operative systems such as data fusion, routing, security and privacy. Eventually, conclusions are drawn.

\section{Architecture}

In co-operative systems the specification of a unified communication architecture plays a central role for further deployment. As a result of the deployment of co-operative systems the road users will benefit from improved safety, reduced traffic congestion, environmental friendly driving and much more. The key to achieving these benefits lies in the specification of a common and standardized communication architecture among the various components of such systems. This architecture comprises four main components which can be composed arbitrarily to form a co-operative intelligent transport system. To form such a system there is no need to have all these four components available but a subset of them is sufficient. The components can communicate with each other either directly within the same communication network or indirectly across several communication networks. These four components are depicted in Figure 2 and are briefly described in the following. For a more detailed description one can refer to (Bechler et al., 2010; ETSI, 2010). 


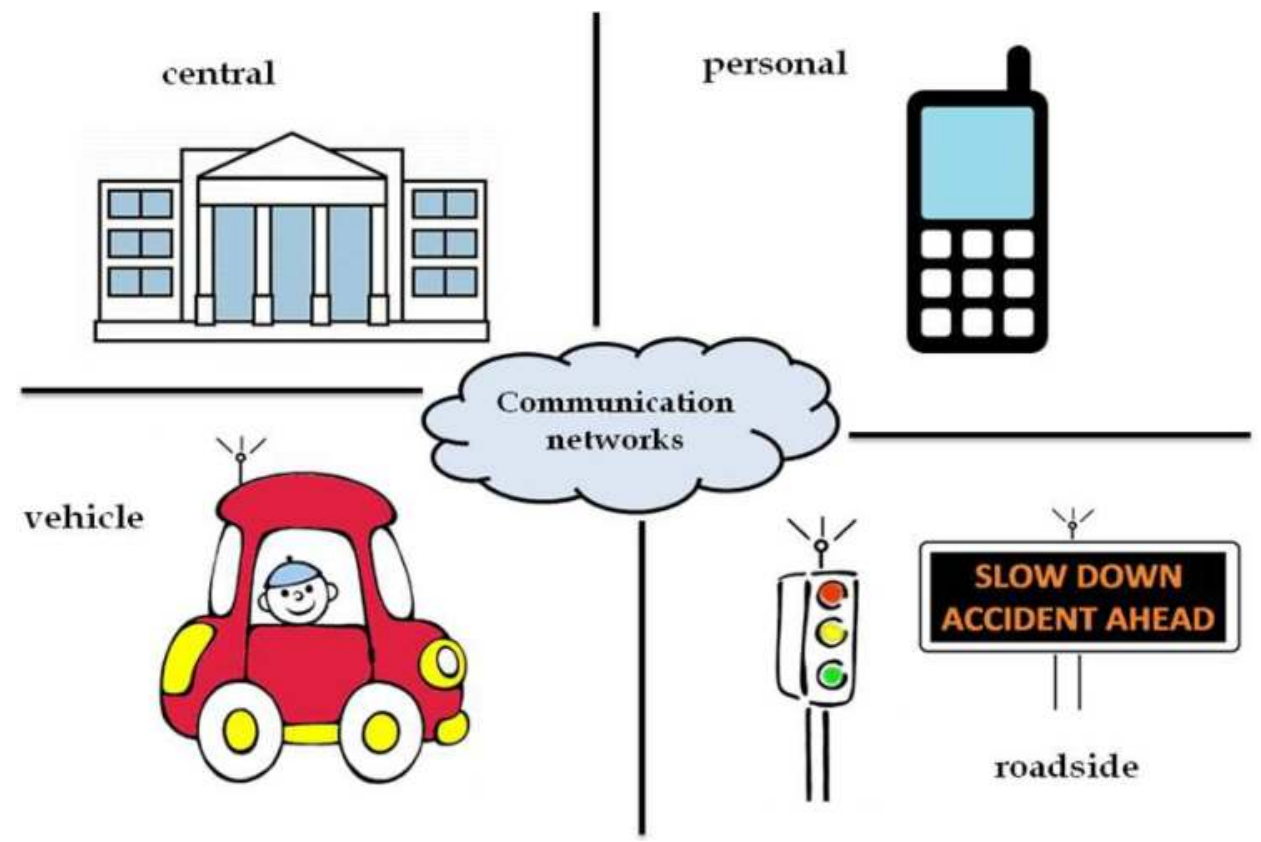

Fig. 2. Communication Architecture Components.

\subsection{Vehicle component}

The vehicle is equipped with communication capabilities (i.e. a router, embedded PCs) to establish communication with other vehicles and the roadside infrastructure. These modules have access to the CAN network of the vehicle as well as to other vehicle data which they collect, process and communicate to other vehicles, the roadside units or the central system. The exact HW solution is not strictly defined and it can be a unique HW unit or several units which form a LAN inside the vehicle.

\subsection{Roadside component}

The roadside component includes variable message signs, traffic lights and other units which are equipped with communication capabilities. This way, the roadside component can communicate with other vehicles by sending them information or acting as a relay station supporting multi-hop communication. Moreover, this component can communicate with other roadside units and the central system and therefore forward information received from vehicles. The roadside component can be also connected to the Internet.

\subsection{Personal component}

The personal component is actually a nomadic device, that is a personal navigator or a smartphone, which can host a variety of ITS applications. These devices can also support cooperative ITS applications based on communication with other road users or the road infrastructure. 


\subsection{Central component}

The central component is a public authority or a road operator who manages the cooperative applications or services. An example of such component is a traffic management center which uses roadside units to inform the drivers about traffic status or accidents in a specific road network and suggests alternative routes. The central component can receive information from vehicles or roadside units and in turn send information to them.

\subsection{Reference protocol stack}

Each one of the above components contains an ITS station which in turn comprises a number of ITS specific functions and a set of devices implementing these functions. From a communication's point of view an ITS station is based on the reference protocol stack depicted in Figure 3. This protocol stack follows the ISO/OSI reference model and consists of four horizontal layers and two vertical ones that flank the horizontal stack. Access, networking and transport, facilities and applications layers are the horizontal ones, whereas management and security are the vertical ones.

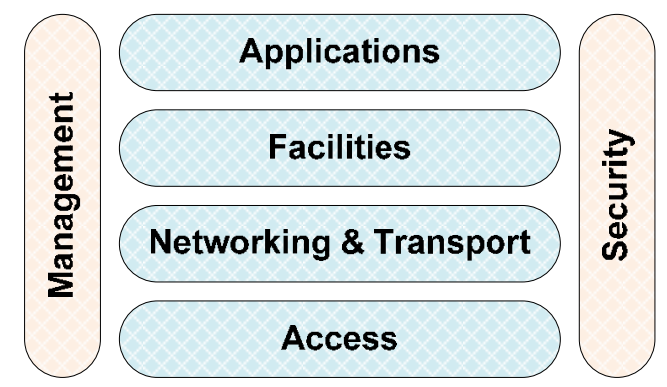

Fig. 3. Reference protocol stack of an ITS station.

\section{Wireless technologies}

The wireless technologies used for the continuous communication among different vehicles and between vehicles and the road infrastructure are the cornerstone of co-operative systems. These technologies concern the networking $\mathcal{E}$ transport and access layers of the reference protocol stack of an ITS station (Figure 3) and can be divided into two main categories: general and vehicular specific communication technologies.

Therefore, for the connection among vehicles and the road infrastructure a mixture of general and vehicular specific technologies is needed. Some of these technologies are already in use, while some others are still under development.

\subsection{General communication technologies}

This category comprises well known wireless communication technologies such as cellular networks, WiMAX, WiFi, infrared, bluetooth, DVB/DAB etc. which are not specifically developed for vehicular networks but play a significant role for future deployment of cooperative systems. Currently, in ITS the main focus is on cellular networks and WiMAX and for this reason only those two will be analyzed below. 


\subsubsection{Cellular networks}

Cellular networks are evolving rapidly to support the increasing demands of mobile networking. Although these networks are designed for voice data exchange they can be applied also to vehicular networks especially for information and entertainment applications. Nowadays, cellular networks migrate from GPRS, to UMTS, to LTE standards, increasing bandwidth and reducing delay times making these networks appropriate also for other kind of applications such as efficiency and trip planning.

Cellular networks have several characteristics suitable for co-operative systems like large scale usage and long range communication. However, some drawbacks of cellular networks which are relevant to vehicular connectivity are summarized below:

- Increased latency (i.e. voice data higher priority than text data, data sent via base stations)

- No broadcasting capabilities, only support of point-to-point communication

- Operation fees (e.g. internet access, roaming)

However, despite all the above disadvantages, cellular networks can be used for ITS applications which require moderate delay, long range communication, and low data rate. With the migration from $3 G$ towards $4 G$ (such as LTE) the focus remains on technologies that can serve a circular area with Internet connectivity, with no special provision for following the road infrastructure and optimising for connected car services.

\subsubsection{WiMAX}

WiMAX (Worldwide Interoperability for Microwave Access) is based on IEEE 802.16 standard and aims at providing wireless data over long distances in a variety of ways, from point-to-point links to full mobile cellular type access. WiMAX provides support for mobility and it will fill the gap between $3 \mathrm{G}$ and WLAN standards. It offers high data rates $(<40 \mathrm{Mb} / \mathrm{sec})$, portable connectivity at low speeds $(<60 \mathrm{~km} / \mathrm{h})$ and wide area coverage $(<10 \mathrm{~km})$ required to deliver high speed internet access to mobile clients. WiMAX provides a wireless alternative to cable and XDSL for last mile broadband access and can be used for V2I or I2I long range communication. At this point it should be mentioned that WiMAX supports several service levels including guaranteed Quality of Service (QoS) for delay sensitive applications and an intermediate QoS level for delay tolerant applications that require a minimum guaranteed data rate.

\subsection{Vehicular specific communication technologies}

This category includes communication technologies which are dedicated to vehicular applications and actually were the result of additional communication requirements posed by ITS applications. Dedicated communication standards are in development for cooperative systems. At the access layer, a convergence towards the IEEE 802.11p standard can be observed, while standardisation on the network and transport layer is still in progress. Several prototype implementations exist and are used in demonstrations and pilots. IP communication (focus is on IPv6) can be used on top of $802.11 \mathrm{p}$, but due to the highly dynamic character of the network (i.e. movement of vehicles, relatively short communication distances) dedicated standards have been developed and are being 
standardised by ISO and ETSI. The most important of them, namely DSRC, WAVE and CALM, are illustrated below.

\subsubsection{Dedicated Short Range Communications}

Dedicated Short Range Communications (DSRC) is a short to medium range communications service that supports both public safety and private operations in $\mathrm{V} 2 \mathrm{~V}$ and V2I communication environments (DSRC, 2003). DSRC is meant to be a complement to cellular communications by providing very high data transfer rates in circumstances where minimizing latency in the communication link and isolating relatively small communication zones are important.

DSRC is designed for vehicular wireless communications and operates on radio frequencies in the 5.725 to $5.875 \mathrm{GHz}$ (Industrial, Scientific and Medical - ISM) band in Europe and in the 5.850 to $5.925 \mathrm{GHz}$ band in the United States. DSRC systems consist of Road Side Units (RSUs) and On Board Units (OBUs) with transceivers and transponders. The DSRC standards specify the operational frequencies and system bandwidths, but also allow for optional frequencies which are covered (within Europe) by national regulations.

The range of communication using DSRC is up to $1000 \mathrm{~m}$ with data rates of $6-27 \mathrm{Mb} / \mathrm{s}$, where vehicles may be moving at speeds up to $140 \mathrm{~km} / \mathrm{h}$. As mentioned previously, DSRC is divided into two types of communication, namely V2V and V2I. V2V communication is used when vehicles need to exchange data among themselves in order for co-operative applications to work properly, whereas V2I communication is used when roadside units are part of the co-operative application. In co-operative systems, some applications are required to send messages periodically (e.g. every $100 \mathrm{~ms}$ ), whereas other applications send messages when an event occurs.

At this point it should be highlighted that DSRC systems are used in the majority of European Union countries, but these systems are currently not totally compatible. Therefore, standardization is essential in order to ensure pan-European interoperability, particularly for applications such as electronic fee collection, for which the European Union imposes a need for interoperability of systems.

Standardization will also assist with the provision and promotion of additional services using DSRC, and help ensure compatibility and interoperability within a multi-vendor environment.

\subsubsection{Wireless Access in Vehicular Environments}

The design of an efficient communication protocol in the automotive sector that deals with privacy, security, multi-channel operation and management of resources is a difficult task, which is under intensive scientific investigation. This task is assigned to a special IEEE working group and the ongoing suite of protocols is the IEEE 1609, mostly known as Wireless Access in Vehicular Environments or simply WAVE (WAVE, 2007).

The WAVE standards define an architecture and a complementary, standardized set of services and interfaces that collectively enable secure V2V and V2I wireless communications. Together these standards provide the foundation for a broad range of 
applications in the transportation environment, including vehicle safety, automated tolling, enhanced navigation, traffic management and many others.

The architecture, interfaces and messages defined in WAVE support the operation of secure wireless communications among vehicles and between vehicles and the road infrastructure. Applications can use these standards in conjunction with equipment operating at $5.9 \mathrm{GHz}$ to provide, for example, services for drivers, road operators, facilities operators and maintenance staff.

The IEEE 1609 Family of Standards for WAVE consists of four trial use standards which have full use drafts under development and two unpublished standards under development:

- IEEE P1609.0 - Draft Standard for Wireless Access in Vehicular Environments (WAVE) Architecture

This standard describes the WAVE architecture and services necessary for multichannel DSRC/WAVE devices to communicate in a mobile vehicular environment.

- IEEE 1609.1-2006 - Trial Use Standard for Wireless Access in Vehicular Environments (WAVE) - Resource Manager

This standard specifies the services and interfaces of the WAVE Resource Manager application. It describes the data and management services offered within the WAVE architecture. It defines command message formats and the appropriate responses to those messages, data storage formats that must be used by applications to communicate between architecture components, and status and request message formats.

- IEEE 1609.2-2006 - Trial Use Standard for Wireless Access in Vehicular Environments (WAVE) - Security Services for Applications and Management Messages

This standard defines secure message formats and processing. This standard also defines the circumstances for using secure message exchanges and how those messages should be processed based upon the purpose of the exchange.

- IEEE 1609.3-2007 - Trial Use Standard for Wireless Access in Vehicular Environments (WAVE) - Networking Services

This standard defines network and transport layer services, including addressing and routing, in support of secure WAVE data exchange. It also defines WAVE Short Messages, providing an efficient WAVE-specific alternative to IPv6 (Internet Protocol version 6) that can be directly supported by applications. Further, this standard defines the Management Information Base (MIB) for the WAVE protocol stack.

- IEEE 1609.4-2006 - Trial Use Standard for Wireless Access in Vehicular Environments (WAVE) - Multi-Channel Operations

This standard provides enhancements to the IEEE 802.11 Media Access Control (MAC) to support WAVE operations.

- IEEE P1609.11 Over-the-Air Data Exchange Protocol for Intelligent Transportation Systems (ITS)

This standard will define the services and secure message formats necessary to support secure electronic payments.

Additionally, the IEEE 1609 standards rely on IEEE P802.11p. This proposed standard specifies the extensions to IEEE 802.11 that are necessary to provide wireless communications in a vehicular environment. 


\subsubsection{Communications Access for Land Mobiles}

The Communications Access for Land Mobiles (CALM) framework is an ISO TC204 initiative that specifies a common architecture, network protocols and communication interface definitions for wired and wireless communications using various access technologies including cellular 2nd generation, cellular 3rd generation, satellite, infra-red, 5 $\mathrm{GHz}$ micro-wave, $60 \mathrm{GHz}$ millimetre-wave, and mobile wireless broadband (CALM, 2007). These and other access technologies that can be incorporated are designed to provide broadcast, unicast and multicast communications between mobile stations, between mobile and fixed stations and between fixed stations in the ITS sector.

The CALM concept is therefore developed to provide a layered solution that enables continuous or quasi continuous communications between vehicles and the infrastructure, or between vehicles, using such (multiple) wireless telecommunications media that are available in any particular location, and have the ability to migrate to a different available media where required. Media selection is at the discretion of user determined parameters.

The motivations behind this standardization effort are the following:

- different countries use different ITS media,

- different ITS applications have different requirements, therefore it is impossible to use a single carrier to support all types of applications.

The following communication types are supported by CALM:

- Vehicle-to-Infrastructure: Multipoint communication parameters are automatically negotiated and subsequent communication may be initiated by either roadside or vehicle.

- Infrastructure-to-Infrastructure: The communication system may also be used to link fixed points where traditional cabling is undesirable.

- Vehicle-to-Vehicle: A low latency peer-to-peer network with the capability to carry safety related data such as collision avoidance and other vehicle-vehicle services such as adhoc networks linking multiple vehicles.

At a high level, on the one side there are multiple services possibly operating simultaneously all requesting communications services, whereas on the other side there is a possibility of multiple communications media opportunities in the vehicle to handle the transaction. In the middle CALM is located managing quasi continuous communications using the available media, to satisfy the needs of one or multiple applications. It is important to understand that the vehicle may be maintaining multiple simultaneous sessions.

Finally, it is important to highlight that the specifications and standards of CALM are not a physical piece of equipment. While CALM may indeed operate through a "box" designed to achieve its tasks, it is actually a set of protocols, procedures and management actions. The implementation is actually a commercial decision.

\section{Applications}

Together with the evolution of vehicular networks numerous novel ITS applications have emerged. Typical examples of co-operative applications include remote diagnostics, 
collision avoidance, online navigation, map update, congestion avoidance for the driver, internet in the vehicle for passengers (e.g. gaming, downloading videos, reading the news etc.). These applications can be divided into three major categories, namely safety, efficiency and infotainment. In the following some indicative applications in each category are selected and will be described briefly.

\subsection{Safety}

One of the main goals of transport authorities is the minimization of traffic accidents and the increase of road safety. The exploitation of wireless technologies will be a significant asset towards this direction as it has been obvious from the results of the SAFESPOT project (SAFESPOT, 2006-2010). Examples of co-operative safety related applications will be given below.

\subsubsection{Frontal collision warning}

Frontal collisions represent a major proportion of accidents worldwide. Typical causes of such accidents are the distraction of the driver, sudden braking of a vehicle ahead, the presence of a stationary obstacle in front of the vehicle (e.g. right after a turn) etc. Conventional collision warning systems are based on sensors installed in the vehicle. These sensors could be long range radars for adaptive cruise control, camera sensors for objects detection, cameras covering the blind spot area, laser scanners for both detecting and classifying objects. This way a vehicle can be informed about events and targets which are within range of the detection sensors. Figure 4 shows a frontal collision warning application where the vehicle in front brakes while other vehicles are following.

The reliability and accuracy of a conventional collision warning system is based on the number and the type of sensors used, as well as the type of the environment (i.e. urban, inter-urban, highway) around the vehicle. The occlusion of sensors from obstacles, the limited range of sensors and other physical constraints, reduce system's range and degrade its performance. Apart from these factors, a collision warning system to function properly needs a multitude of sensors to cover the entire area around the vehicle, which makes such a system extremely expensive.

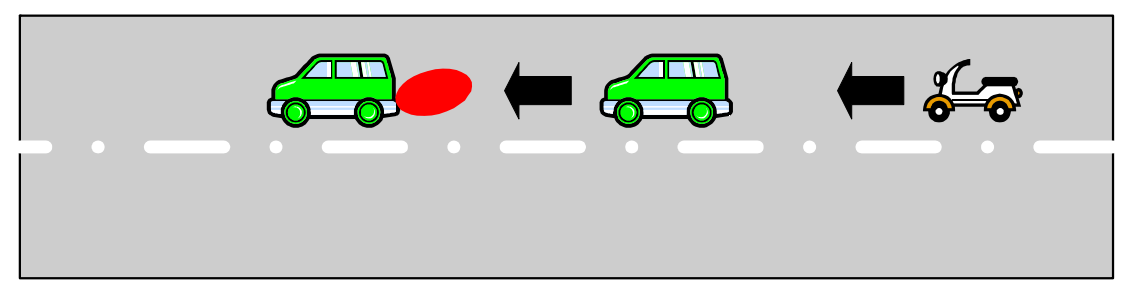

Fig. 4. Frontal collision warning application.

The collision warning system can be much more effective if other neighboring vehicles communicate with the subject vehicle, extending thus the perception of the driver in relation to the limited perception based only on sensors installed in the vehicle. Actually, this is the principle of co-operative collision warning systems. While driving, equipped vehicles 
anonymously share relevant information, including their position, speed and direction. This way each vehicle monitors the intentions of other drivers and the location and behavior of all vehicles in the neighborhood. When a vehicle detects a critical situation, the system warns the driver with a visual, audible and/or haptic manner. Thus, the driver has enough time to intervene and avoid a collision.

In time critical situations, immediate intervention to avoid a collision is feasible with the use of communication. This would not be possible in case only onboard sensors were used because of the delay in detecting and classifying objects and analyzing the ongoing situation. The co-operative approach also has great influence on the classification of objects. If vehicles are equipped with wireless communication they can directly exchange information about their type (e.g. truck, car, motorbike).

\subsubsection{Intersection safety}

A significant number of accidents in urban areas occur at intersections. The reasons for this are the significant burden of the driver from the complex situations that can occur at intersections due to many vehicles that are flooding them from different directions, the variety of road users (cars, trucks, pedestrians, cyclists etc.) as well as buildings and walls that limit the visibility of the driver.

In order for an accident or a dangerous situation to occur at an intersection it is supposed that there should be a violation of traffic rules such as traffic light or "STOP" sign violation. But intersections are complex road environments and accidents are likely to occur even if the rules are obeyed (e.g. abrupt braking while the traffic light turns from green to red). A simplified example of an intersection safety application is shown in Figure 5.

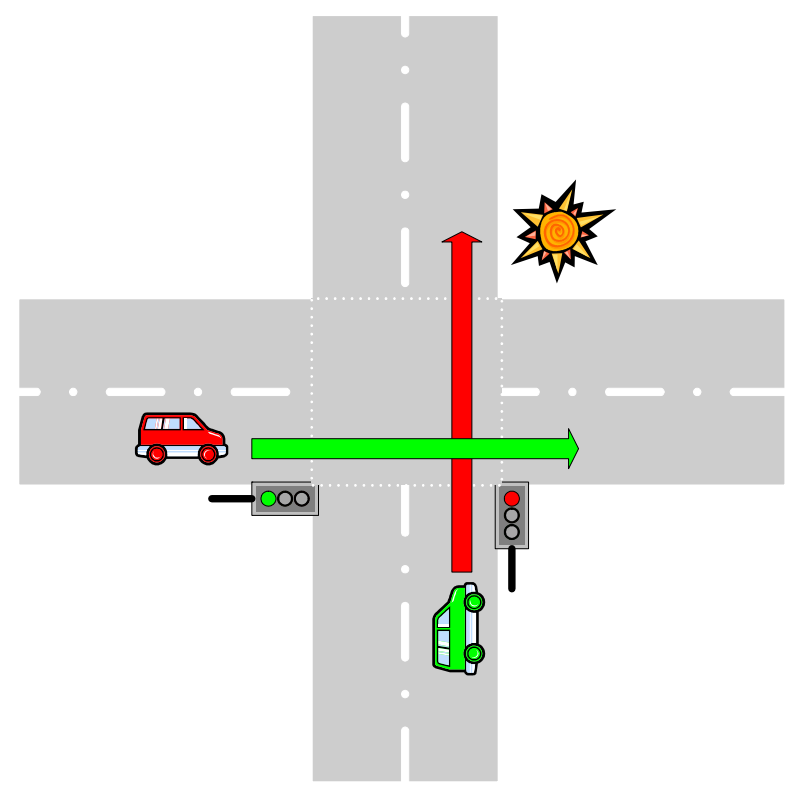

Fig. 5. Intersection safety application. 
The benefits from the exploitation of wireless communication for safety reasons at intersections are significant. First of all, the traffic lights and other traffic signs can emit their status, together with information concerning the instant that this status is going to change (e.g. traffic light that turns from red to green), to the interested drivers informing them about the real ongoing situation at the intersection. In addition, vehicles can emit their position and dynamic state (speed, acceleration, steering angle) and thus to inform other drivers at the intersection about their presence. Otherwise it would be impossible for other drivers to be aware of their presence because of the occlusion of the surrounding buildings, walls and other obstacles. Finally, some sensors, such as laser scanners, could be installed at critical points at an intersection to detect pedestrians and cyclists (vulnerable road users) and inform the drivers for their presence through wireless communication.

\subsubsection{Slippery road detection}

This application informs the driver about the status of a road segment where there is a possible risk. The risk is primarily related to a slippery road surface that may be due to adverse weather conditions (e.g. ice, rain) or to any extraordinary event (e.g. an oil leak of the vehicle ahead). The detection of the slippery road segment can be carried out by a vehicle either directly by specific sensors or indirectly by activation of the ABS or ESP system. Even though this information, about the dangerousness of the road, is transmitted to the driver directly, it may be too late to take action because it is highly likely that the vehicle has slipped already since the ESP or ABS has been activated. A slippery road detection application is depicted in Figure 6.

As it is obvious, this application has almost no interest without the use of wireless communication. By taking advantage of wireless communication the vehicular network can share information related to hazardous road segments such as slippery roads. For example, a vehicle that its ESP system is activated associates this data with its current location and notifies other nearby vehicles and possibly a RSU if it is in communication range. The neighboring vehicles receiving such information shall promptly inform the drivers about the potential risk, while retransmitting this information to other nearby vehicles (multi-hop communication). As an alternative, a RSU can be equipped with some special sensors which can detect the hazardous road conditions and inform the drivers approaching this area.

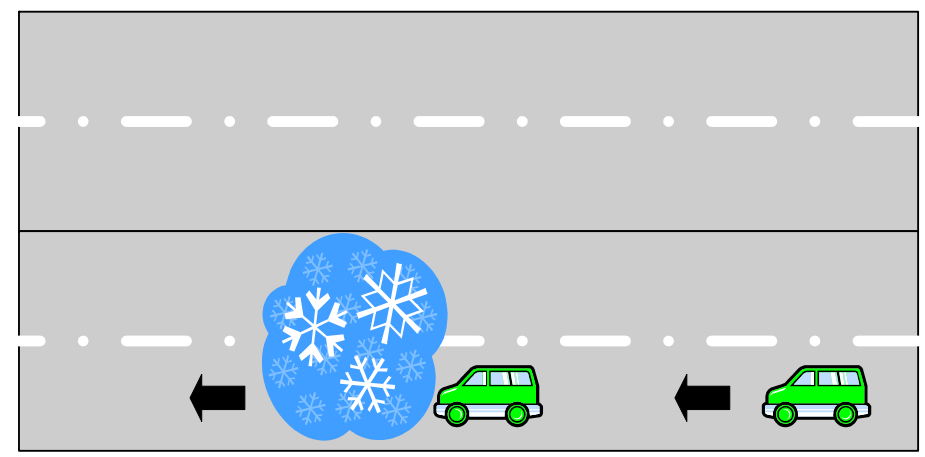

Fig. 6. Slippery road detection application. 
As a conclusion, there is an apparent advantage in using wireless communication to broadcast information about the road conditions to the approaching drivers who will then have sufficient time to react. With this co-operative approach the RSU has the ability to transmit road condition information to the traffic management center which then can be analyzed and checked for accuracy and quality and transmitted to other vehicles that drive on this road segment.

\subsection{Efficiency}

The climate change that has been observed in recent years has also affected the priorities in the transport agenda. Nowadays minimization of $\mathrm{CO}_{2}$ emissions related to transport and environmental friendly driving comprises a top priority. The results of the CVIS project (CVIS, 2006-2010) have shown the added benefit from the use of wireless communication to enhance efficiency in transport. Examples of co-operative efficiency related applications will be highlighted in the following.

\subsubsection{Enhanced route planning}

In this application the infrastructure continuously collects information related to traffic density and makes forecasts for road segments with potential traffic jams. Then when an equipped vehicle drives next to a RSU the traffic density information on the neighboring area as well as driving instructions are transmitted to it. This information is processed by the vehicle and then the driver is informed about possible delays and alternative routes to avoid traffic jams. This way a significant number of drivers could be guided around congested areas so the entire transport system to become more efficient. A significant side effect of this application will be the reduction of pollution deriving from vehicles got stack in congested highways.

Figure 7 shows an enhanced route planning scenario in which the RSU informs the driver of the red vehicle about heavy traffic ahead and suggests a faster alternative route to the driver's final destination. It is obvious from the description of this application that for its implementation a RSU with wireless communication is essential to provide the necessary information. A vehicle equipped only with some perception sensors, without communication capabilities, could make a rough estimation about the traffic density in the road segment it is currently driving, but without any information on alternative routes.

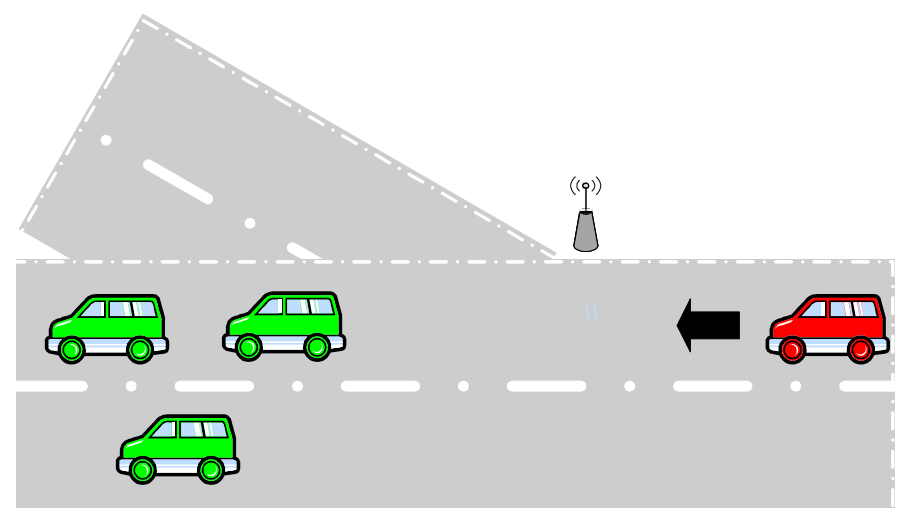

Fig. 7. Enhanced route planning application. 


\subsubsection{Optimal speed advice}

The aim of this application is to provide the needed information to the driver in an effort to make driving smoother and reduce significantly start and stop situations. As the vehicle approaches a signalized intersection it receives information about the exact position of the traffic light and the duration of its current status (e.g. the remaining seconds for the traffic light to become red).

Based on this information, that is using the distance from the intersection and the time until the traffic light turns to green, the approaching vehicle calculates the speed that it should follow to avoid stopping at the intersection. Then this "optimal" speed is provided to the driver and if this suggestion is followed it is very likely that the traffic light will turn into green as soon as the vehicle reaches the intersection and there is no need for it to stop. Fewer stops result in increased traffic flow and reduced fuel consumption for the equipped vehicles.

Figure 8 shows an example of this application in which the driver follows the optimal speed suggestion and thus will not have to stop at the intersection. For such kind of application to become a reality it is essential that the traffic light should be equipped with wireless communication capabilities.

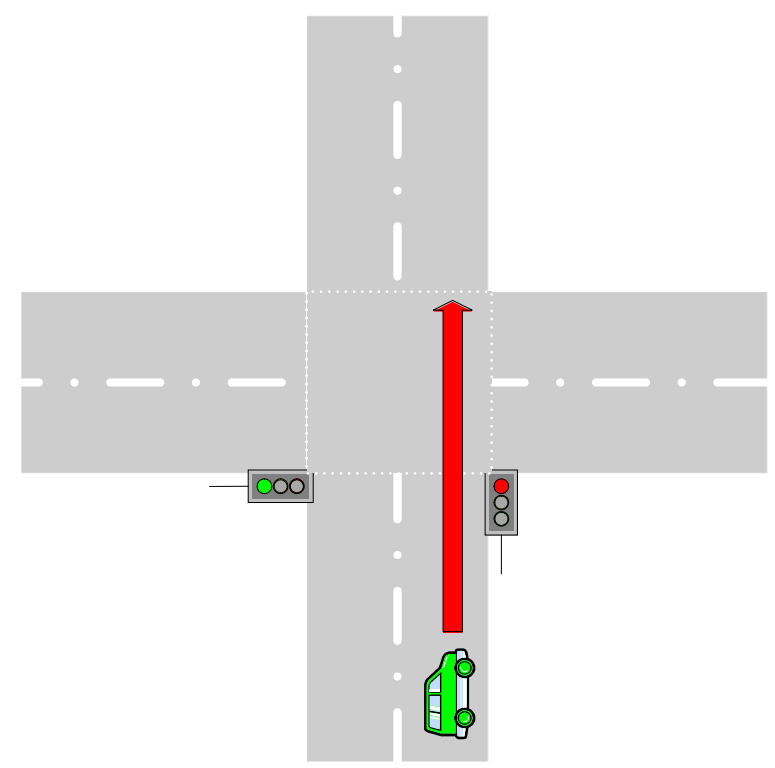

Fig. 8. Optimal speed advice application.

\subsubsection{Traffic merge assistant}

The traffic merge assistant application allows the merging of cars in a joint traffic flow without the need to interrupt their smooth flow. When a vehicle enters a highway from an entrance communicates this intention to the neighboring vehicles. The vehicle entering the 
highway requires specific maneuvers by surrounding vehicles to adapt in a safe and continuous way to the traffic. If there are no objections from other drivers, then either the traffic will be adjusted automatically or advice will be given to the drivers on how to act. In this way the vehicle entering the highway can adjust smoothly into the flow of traffic without causing major disruptions to it. This application can be enhanced by using a RSU which can determine the movements of each participant.

This application, as shown in Figure 9, requires a significant number of vehicles to be equipped with wireless capabilities. Moreover, it is one of the most difficult and complex applications which is based on the harmonious and trustworthy co-operation among highly dynamic vehicles.

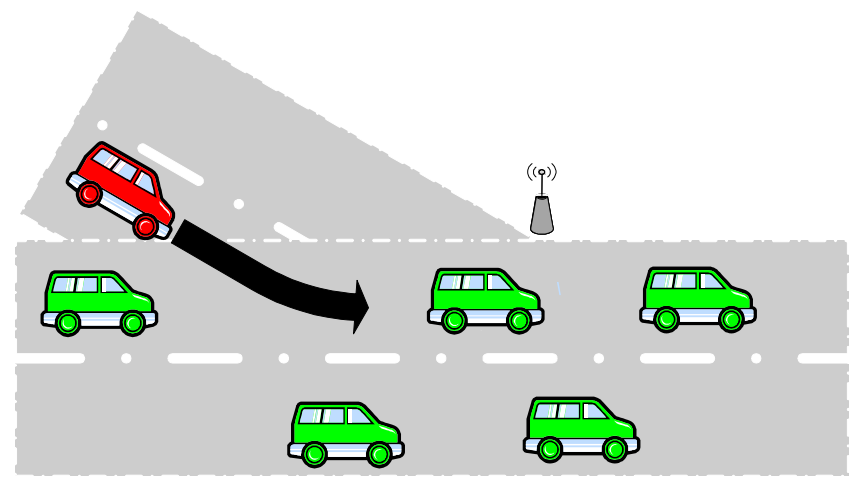

Fig. 9. Traffic merge assistant application.

\subsection{Infotainment}

The entertainment and information provision to the driver and the passengers might not be life critical but is still very important in today's society, where internet and exchange of information dominate. Examples of co-operative applications related to information and entertainment (infotainment) will be outlined in this section.

\subsubsection{Points of interest}

The Points of Interest (PoI) application allows local businesses, touristic attractions, restaurants, gas stations and so on to advertise their availability to nearby vehicles. In this case, a RSU transmits information about a PoI, such as its location, hours of operation and pricing. Then this information is filtered by the vehicles dynamically, depending on the case, and the relevant information is presented to the driver. For example, if the fuel level is low, the vehicle could show to the driver the locations and prices of gas stations in the surrounding area. The benefit of this application is that advertising gets more effective as the driver moves within the geographical area where the PoI is located and it is more likely to visit it rather than if he listened about it to a radio station or found it on the web. Moreover, another benefit is that consumers receive up to date information directly from a business in the neighborhood. 


\subsubsection{Internet access}

This application allows drivers and passengers to access the Internet. This in turn means the use of all types of services based on the IP protocol inside the vehicle. Therefore, a multi-hop route from a RSU to the relevant vehicle is installed and maintained to act as a gateway to the Internet. This path of multiple hops takes place transparently to the upper layers of the protocol stack and enables almost any service based on IP protocol to be used inside vehicles. Finally, this application allows access to the driver and passengers in any type of information available on the Internet (e.g. downloading of updated digital maps).

\subsubsection{Remote diagnostics}

This application allows an authorized service station to assess the condition of a vehicle without needing a physical connection with it. When a vehicle enters the parking building of the station, remote diagnostics system may ask the vehicle about relevant information to support the diagnosis of the problem reported by the client. Moreover, as the vehicle is approaching its service history and the necessary customer information can be retrieved from a database and be ready for use by the technician. If software updates are necessary, the system can install the updates without a physical connection. This application can reduce the amount of time required for a customer during a visit to an authorized service station. This fact will also reduce both the repair cost and the waiting time for the customers.

\section{Research topics}

Co-operative systems have received particular attention, with respect to the research activities in the field of ITS, the last decade. The recent advances in information and communication technologies have enabled the deployment of co-operative systems as an exciting platform for developing new and useful vehicular applications. The research activities focus mainly on data fusion, routing as well as on privacy and security issues which will be analyzed in the following.

\subsection{Data fusion}

Data fusion plays an important role in co-operative systems. A stand alone sensor or several sensors installed in a vehicle cannot overcome certain physical limitations as, for example, the limited range and field of view. Therefore combing information coming from both onboard sensors and wireless messages, encompassing information from other vehicles, broadens the awareness of the driver and increases the reliability of the whole system in case of sensor failure. However, fusing information from highly mobile vehicles, forming a wireless network, is a challenging task (Ahlers \& Stimming, 2008; Lytrivis et al., 2008).

The Joint Directors of Laboratories (JDL) functional model, which is the most prevalent in data fusion community, is depicted in Figure 10. According to this model the data processing is divided to the following levels: signal, object, situation and application. All these levels communicate and exchange data through a storage and system manager (Liggins et al., 2008). 


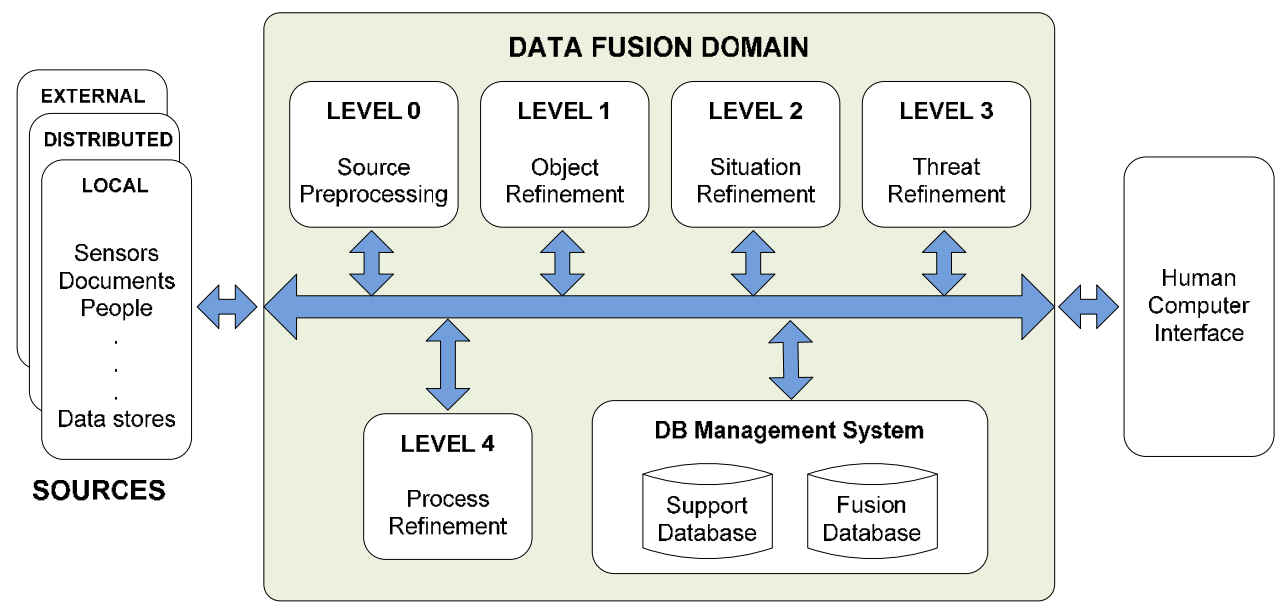

Fig. 10. Joint Directors of Laboratories (JDL) model.

In the data fusion process the main focus is on object and situation refinement levels, which refer to the state estimation of objects and the relations among them, correspondingly. The discrimination between these levels is also made by using the terms low and high level fusion instead of object and situation refinement. The different levels of the JDL model are summarized below:

- Level 0: Preprocessing of sensor measurements (pixel/signal-level processing).

- Level 1: Estimation and prediction of entity states on the basis of inferences from observations.

- Level 2: Estimation and prediction of entity states on the basis of inferred relations among entities.

- Level 3: Estimation and prediction of effects on situations of planned or estimated/predicted actions by the participants.

- Level 4: Adaptive data acquisition and processing related to resource management and process refinement.

In the past decade the advances in autonomous sensor technologies and the major objective of the European Union to reduce to a half road accidents and fatalities by 2010, led to the development of advanced driver assistance systems (ADAS). The fusion of data coming from different advanced in-vehicle sensors was initially in the centre of this attempt. However, this approach suffers from serious limitations. Specifically:

- the perception environment of the vehicle cannot go beyond the sensing range,

- the sensor systems cannot perform well in all environments (the urban roads comprise a major challenge),

- in several cases the system is not able to perceive the situation in time in order to warn the driver and suggest a corrective action,

- the cost of the sensor systems is too high and so their installation is feasible only at luxurious vehicles. 
Recently the focus of research activities on co-operative systems is driven by the attempts to overcome all the above limitations. The limited bandwidth, security issues, privacy, reliability and propagation are some of the emerging disadvantages of the wireless connectivity in vehicles. All these issues poses additional challenges to the data fusion process. The association and synchronization of data from on-board sensors together with the wireless network data is the main challenge. Moreover, the manipulation of delayed information and the reliability of the information transferred via the network are other important issues.

\subsection{Routing}

Routing is the process of finding a path from a source node to a destination node. In this section the word "node" will be used interchangeably with the word "vehicle" because a vehicle is actually a node of a vehicular network. Since each node has limited transmission range, messages often need to be forwarded by other nodes in the network to reach their final destination (i.e. multi-hop communication).

Despite the fact that there are already some routing protocols available, which are mainly derived from the Mobile Ad-hoc Network (MANET) domain, it is an intensive scientific research area due to the highly dynamic nature of vehicular networks.

The routing protocols designed specifically for co-operative systems (Lee et al., 2010; Li \& Wang, 2007) can be divided into two broad categories: topology-based routing and locationbased routing. The former use information about the existing links of the network to forward the relevant messages. In the latter forwarding decisions are based on the location of the nodes. Moreover, position based routing protocols can be further divided into proactive and reactive.

Proactive algorithms are using classical routing strategies such as distance-vector routing or link-state routing. Proactive algorithms maintain routing information about the available paths in the network even if these paths are not currently used. The main disadvantage of this approach is that the maintenance of unused paths occupies a significant part of the available bandwidth if the network topology changes frequently.

In response to the problem of maintaining the paths of proactive protocols, reactive routing protocols were created. Reactive protocols maintain only routes that are in use, thereby reducing the load on the network when only a small subset of available paths are used.

In location-based routing, forwarding decisions are based on the location of the node that forwards the message according to the location of the source and destination nodes. In contrast to pure ad hoc approaches which are based on topology-based routing, here it is not necessary to setup or maintain a path since packets are forwarded directly. Location-based routing protocols consist of location services and geographical forwarding.

Geographical forwarding takes advantage of a topological assumption which works well for wireless ad hoc networks: nodes that are physically close are likely to be close in the network topology too. Each node is aware of its location using technologies such as GPS and periodically broadcasts its presence, location and speed to its neighbors. Thus, each node maintains a table with the identities and locations of its current neighbors. When one node 
needs to forward a packet it includes the identifier of the destination-node and its geographical location into the header of the packet. Each node along the forwarding path consults its list of neighbors and forwards the packet to the neighbor closest to the destination in terms of physical location, until it reaches its final destination.

Although the geographical forwarding works well for networks where nodes are uniformly distributed, perhaps cannot find a route to a packet's destination when the packet has to travel around a topology "hole" - that is, when an intermediate forwarding node has no neighbors who are closer than itself to the destination of the packet.

An overview of some topology-based routing algorithms is given below:

- Ad Hoc On Demand Distance Vector (AODV) is a routing algorithm where the nodes of the network upon receiving a broadcast query they record the address of the querying node to their routing table. The process of recording the previous hop is called backward learning. When a packet reaches its destination a reply packet is sent back to the source through the full path retrieved from the process of backward learning. At every node of the path, the previous hop should be recorded, creating this way the forward path from the source. The query together with the response create a complete bidirectional path. After setting the path, it is maintained as long as the source uses it. A failure on a link will be reported recursively to the source and in turn this will trigger another query-response process for finding the new route. More details about AODV one can find in (Perkins \& Royer, 1999).

- Dynamic Source Routing (DSR) is an algorithm that uses source routing, that is the source indicates to a data packet the sequence of intermediate nodes on the routing path. In DSR, the query packet copies in its header the identities of the intermediate nodes it has already visited. Afterwards, the destination uses the query packet to retrieve the entire path to respond to the source. As a result, the source can establish a path to the destination. If the destination node is allowed to send multiple routes responses, the source node may receive and store these multiple routes. An alternative route can be used in case a link of the current path is broken. In a low mobility network DSR has the advantage over AODV in case the alternative route can be tested before the DSR initiates another query to discover the route. There are two major differences between AODV and DSR. The first is that in AODV data packets carry the destination address, while in DSR data packets carry all the routing information. This means that DSR has probably more routing burden than AODV. Moreover, as the diameter of the network increases, the burden on the data packet will continue growing. The second difference is that in AODV route response packets carry the destination address and the sequence number, while in DSR they carry the address of each node along the route. The interested reader in DSR can refer to (Johnson \& Maltz, 1996).

A brief description of some location-based routing algorithms is given below:

- Connectivity-Aware Routing (CAR) is a routing algorithm which derives from the work performed by the Preferred Group Broadcast (PGB) to reduce the broadcasted packets during the discovery of the AODV route taking also into account the mobility of the nodes. CAR uses the route discovery of AODV to find routes with reduced broadcasting from PGB. However, the nodes forming the route record neither their previous node from the backward learning nor their previous node which forwards 
the response route packet from the destination. Only anchor points, which are nodes near an intersection or a curve of the road, are recorded in the route discovery packet. A node defines itself as an anchor point if its velocity vector is not parallel to the velocity vector of the previous node in the packet. The destination may receive multiple route discovery packets. If this happens it chooses the path that provides the best connectivity and the shortest delays. More details about CAR can be found in (Naumov \& Gross, 2007).

- Geographic Source Routing (GSR) is based on the availability of a map. It calculates the shortest Dijkstra path of the cascading graph where vertices are intersection nodes and edges are the roads connecting these vertices. The sequence of intersections is setting up the route to the destination. Then the packets are greedily forwarded between intersections. GSR does not take into account the connectivity between two intersections, so the route might not be fully connected. In case such a situation occurs a recovery with greedy forwarding takes place. The most significant difference between the GSR and CAR is that CAR does not use a map and uses proactive discovery of anchor points that indicate a turn at an intersection. More details about GSR can be found in (Lochert et al., 2003).

\subsection{Security and privacy}

Security in V2V and V2I communications is a prerequisite for future development of cooperative systems and actual deployment in the real world. Co-operative systems have to ensure that data transmission derives from a trusted source and has not been counterfeited. For example, in a red light violation warning application, the in-vehicle system receives data from the equipment which is installed in the traffic light and then decides to issue or not a warning to the driver. An incorrect transmission from a malfunctioning or compromised unit might jeopardize vehicle's safety as well as others' safety in the vicinity. Similarly, the future development of safety applications is jeopardized without securing that transmissions are coming from a trusted source.

Privacy and anonymity are primary issues that also have to be addressed. In co-operative applications vehicles are broadcasting messages about their current location, speed and heading. It is desirable for the users to maintain their privacy since they fear that such a system could be used to build tracking mechanisms which would allow harassment, automatic issue of tickets for speeding or otherwise act in an undesirable way for them.

Unfortunately, on the other hand anonymity may be abused. Some examples are sending fake information or spamming. If the system ensures accountability ${ }^{1}$ then the users know that there will be consequences for others if their data is abused. The challenge here is ensuring anonymity and at the same time accountability, as they seem to be conflicting.

There are many ongoing research activities on security and privacy in co-operative systems. Some ideas that have been proposed for solving such issues include public key certificates or digital signatures. For more information the interested reader can refer to (Fischer et al., 2007; Raya \& Hubaux, 2007).

${ }^{1}$ Accountability is the ability to attribute actions to the entity that caused those actions. 


\section{Conclusion}

An overview of co-operative systems and their importance in future transportation systems has been presented in this chapter. The chapter started with a short introduction about the historical background and the purpose of co-operative systems. Emphasis was given to the communication architecture, including its components, which is mainly the outcome of the efforts carried out so far in Europe. Also the importance of the definition of a common architecture for further deployment of co-operative systems was stressed.

In the following, the focus was on the wireless technologies used within the co-operative systems framework which are divided into two categories: general and vehicular specific communication technologies. These technologies are the cornerstone of co-operative systems and their objective is the continuous communication among different road users (vehicles, motorbikes, trucks, roadside units, infrastructure etc.). To achieve this continuous and seamless communication a mixture of general and vehicular specific technologies is needed. Some of these technologies are already in use, while some others are still under development.

Additionally, some co-operative applications were described which are categorized into three main groups: safety, efficiency and infotainment. The applications addressing safety and efficiency are of great importance today because the minimization of accidents and their consequences as well as the reduction of $\mathrm{CO}_{2}$ emissions are the primary targets worldwide. Finally, emphasis was given on hot research topics concerning co-operative systems such as data fusion, routing, security and privacy. A general description highlighting each of these topics, the research challenges as well as some solutions were indicated.

Although many problems are not yet solved, the general feeling is that vehicles could benefit from evolving wireless communications in the near future, making "talking vehicles" a reality. Co-operative systems will not only provide lifesaving and environmental friendly applications, but they will become a powerful communication tool for their users.

\section{References}

Ahlers, F. \& Stimming, C. (2008). "Cooperative Laserscanner Pre-Data-Fusion", in Proc. IEEE Intelligent Vehicles Symposium (IV 2008), Eindhoven, 2008, pp. 1187-1190

Bechler, M. et al. (2010). European ITS Communication Architecture, Overall Framework, Proof of Concept Implementation, version 3.0, COMeSafety, February 2010

CALM (2007). International Organization for Standardization, Intelligent Transport SystemContinuous Air Interface Long and Medium - Medium Service Access Point, Draft International Standard ISO/DIS 21218, 2007

CVIS (2006-2010). "Cooperative Vehicle-Infrastructure Systems", Integrated Project cofunded by the European Commission, Available from http:/ / www.cvisproject.org

DSRC (2003). Standard Specification for Telecommunications and Information Exchange Between Roadside and Vehicle Systems-5GHz Band Dedicated Short Range Communications (DSRC) Medium Access Control (MAC) and Physical Layer (PHY) Specifications, September 2003 
ETSI (2010). ETSI EN 302665 - Intelligent Transport Systems (ITS): Communications Architecture, v1.1.1, European Standard (Telecommunications series), September 2010

ETSI (2011). European Telecommunications Standards Institute (ETSI), Intelligent Transport Systems - ITS, Available from

http://www.etsi.org/WebSite/Technologies/IntelligentTransportSystems.aspx

Fischer, L.; Stumpf, F. \& Eckert, C. (2007). "Trust, Security and Privacy in VANETs - A Multilayered Security Architecture for C2C-Communication", In VDI/VWGemeinschaftstagung: Automotive Security, Wolfsburg, Germany, November 2007

Hartenstein, H. \& Laberteaux, K. (2010). VANET Vehicular Applications and Inter-Networking Technologies (Intelligent Transport Systems), Wiley, 1st edition, March 2010

Johnson, D. \& Maltz, D. (1996). "Dynamic Source Routing in Ad Hoc Wireless Networks," Mobile Computing, T. Imielinski and H. Korth, Eds., Ch. 5, Kluwer, 1996, pp. 153- 81

Lee, K.; Lee, U. \& Gerla, M. (2010). Survey of Routing Protocols in Vehicular Ad Hoc Networks, Advances in Vehicular Ad-Hoc Networks, M.Watfa Book Editor, IGI Global

Li, F. \& Wang, Y. (2007). "Routing in vehicular ad hoc networks: A survey", IEEE Vehicular Technology Magazine, June 2007, Vol. 2, Issue 2, pp. 12-22

Liggins, M.; Hall, D. \& Llinas J. (2008). Handbook of Multisensor Data Fusion: Theory and Practice, Second Edition, CRC Press

Lochert, C.; Hartenstein, H.; Tian, J.; Fussler, H.; Hermann, D. \& Mauve, M. (2003). “A routing strategy for vehicular ad hoc networks in city environments," IEEE Intelligent Vehicles Symposium 2003, 9-11 June 2003, pp. 156-161

Lytrivis, P.; Thomaidis, G. \& Amditis, A. (2008). "Cooperative Path Prediction in Vehicular Environments," in Proc. 11th Int. IEEE Conf. on Intelligent Transportation Systems (ITSC 2008), Beijing, 2008, pp. 803-808

Naumov, V. \& Gross, T. (2007). "Connectivity-Aware Routing (CAR) in Vehicular Ad-hoc Networks," 26th IEEE International Conference on Computer Communications (INFOCOM 2007), May 2007, pp.1919-1927

Perkins, C. \& Royer, E. (1999). “Ad-Hoc On-Demand Distance Vector Routing," Proc. IEEE WMCSA '99, New Orleans, LA, Feb. 1999, pp. 90-100

Popescu-Zeletin, R.; Radusch, I. \& Rigani, M. A. (2010). Vehicular-2-X Communication: Stateof-the-Art and Research in Mobile Vehicular Ad hoc Networks. Springer, 1st Edition, May 2010

Raya, M. \& Hubaux, J.P. (2007). "Securing vehicular ad hoc networks", Journal of Computer Security 15 (2007), IOS Press, pp. 39-68

SAFESPOT (2006-2010). "Cooperative vehicles and road infrastructure for road safety", Integrated Project co-funded by the European Commission, Available from http:/ / www.safespot-eu.org

WAVE (2007). IEEE Standards Association, IEEE P1609.1 - Standard for Wireless Access in Vehicular Environments (WAVE) - Resource Manager, IEEE P1609.2 - Standard for Wireless Access in Vehicular Environments (WAVE) - Security Services for Applications and Management Messages, IEEE P1609.3 - Standard for Wireless Access in Vehicular Environments (WAVE) - Networking Services, IEEE P1609.4 Standard for Wireless Access in Vehicular Environments (WAVE) - Multi-Channel 
Operations, adopted for trial-use in 2007, IEEE Operations Center, 445 Hoes Lane, Piscataway, NJ, 2007

Zhou, M.-T.; Zhang, Y. \& Yang, L. (2010). Wireless Technologies in Intelligent Transportation Systems (Transportation Issues, Policies and R \& D), Nova Science Pub Inc, June 2010 


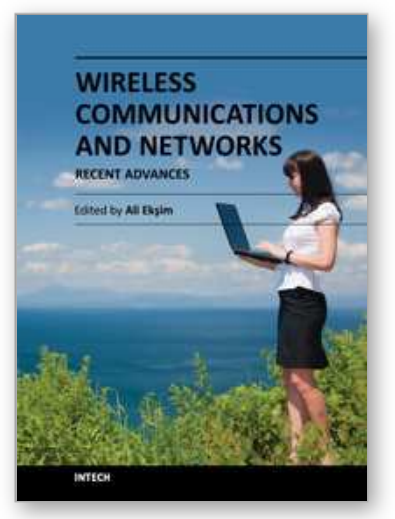

\author{
Wireless Communications and Networks - Recent Advances \\ Edited by Dr. Ali Eksim
}

ISBN 978-953-51-0189-5

Hard cover, 596 pages

Publisher InTech

Published online 14, March, 2012

Published in print edition March, 2012

This book will provide a comprehensive technical guide covering fundamentals, recent advances and open issues in wireless communications and networks to the readers. The objective of the book is to serve as a valuable reference for students, educators, scientists, faculty members, researchers, engineers and research strategists in these rapidly evolving fields and to encourage them to actively explore these broad, exciting and rapidly evolving research areas.

\title{
How to reference
}

In order to correctly reference this scholarly work, feel free to copy and paste the following:

Panagiotis Lytrivis and Angelos Amditis (2012). Intelligent Transport Systems: Co-Operative Systems (Vehicular Communications), Wireless Communications and Networks - Recent Advances, Dr. Ali Eksim (Ed.), ISBN: 978-953-51-0189-5, InTech, Available from: http://www.intechopen.com/books/wirelesscommunications-and-networks-recent-advances/intelligent-transport-systems-co-operative-systems-vehicularcommunications-

\section{INTECH}

open science | open minds

\section{InTech Europe}

University Campus STeP Ri

Slavka Krautzeka 83/A

51000 Rijeka, Croatia

Phone: +385 (51) 770447

Fax: +385 (51) 686166

www.intechopen.com

\section{InTech China}

Unit 405, Office Block, Hotel Equatorial Shanghai

No.65, Yan An Road (West), Shanghai, 200040, China

中国上海市延安西路 65 号上海国际贵都大饭店办公楼 405 单元

Phone: +86-21-62489820

Fax: +86-21-62489821 
(C) 2012 The Author(s). Licensee IntechOpen. This is an open access article distributed under the terms of the Creative Commons Attribution 3.0 License, which permits unrestricted use, distribution, and reproduction in any medium, provided the original work is properly cited. 